\title{
Evaluation and comparison of five experimental BPH/prostate cancer treatment modalities
}

P. J. Hoopes, K. Wishnow, Luanna Bartholomew, E. Jonsson, J. Williams, et al.

P. J. Hoopes D.V.M., K. A. Wishnow, Luanna R. Bartholomew, E. Jonsson, J. Chandler Williams M.D., Karen L. Moodie, Terence Z. Wong M.D., R. D. Harris, Thomas P. Ryan, B. Stuart Trembly, Thomas A. McNicholas, J. A. Heaney, "Evaluation and comparison of five experimental BPH/prostate cancer treatment modalities," Proc. SPIE 10297, Matching the Energy Source to the Clinical Need: A Critical Review, 102970K (24 January 2000); doi: 10.1117/12.375221

SPIE. Event: Digital Optical Computing, 1990, Los Angeles, United States 


\title{
Evaluation and Comparison of Five Experimental BPH/Prostate Cancer Treatment Modalities
}

\author{
P.J. Hoopes ${ }^{* a, b}$, K.A. Wishnow ${ }^{c}$, L.R. Bartholomew ${ }^{a}$, E. Jonsson ${ }^{d}$, \\ J.C. Williams ${ }^{e}$, K.L. Moodie ${ }^{a}$, T.Z. Wong ${ }^{f}$, R.D. Harris ${ }^{\mathrm{g}}$, \\ T.P. Ryan ${ }^{\mathrm{h}}$, B.S. Trembly ${ }^{\mathrm{b}}$, T.A. McNicholas', J.A. Heaney \\ ${ }^{2}$ Dept. Surgery, Dartmouth Medical School, Hanover, NH 03755 \\ ${ }^{b}$ Dept. Bioengineering, Thayer Engineering School/Dartmouth College \\ ${ }^{c}$ N.E. Deconess/Beth Israel Hospital, Boston, MA 02215 \\ ${ }^{\mathrm{d}}$ Dept. Urology, Reykjavik Hospital/Iceland 108 \\ ${ }^{\mathrm{e}}$ Dept. Surgery, University of Florida/Jacksonville 32224 \\ ${ }^{f}$ Dept. Radiology, Duke University Medical Center, Durham, NC 27708 \\ ${ }^{\mathrm{g}}$ Dept. Radiology, Dartmouth Medical School, Hanover, NH 03755 \\ "Ethicon Research \& Development, Somerville, NJ 08876 \\ 'Lister Hospital, Stevenage, UK SW1W8 RH
}

\begin{abstract}
Five non-pharmacological, experimental, prostate (benign hyperplasia/cancer) treatment modalities including transurethral radiofrequency thermotherapy (TURT); transurethral microwave thermotherapy (TUMT); transurethral and transrectal microwave thermotherapy (TUMT/TRMT); interstitial laser coagulation (ILC); and interstitial cryotherapy (IC), are evaluated. These and other similar techniques are currently in various stages of development and clinical use. Most of these modalities produce relatively similar effects in tissue; however, each has pathophysiologic features and potential complications which may preference its use in a specific anatomical and/or disease situation. All treatments were performed using the canine prostate model, by the same investigators. Our studies have shown that although the canine prostate does not respond to injury exactly as the human prostate does, the effects are similar enough to be conceptually, and often specifically, valuable from efficacy and safety standpoints. Two of the five treatments evaluated (TURT, TUMT/TRMT) resulted in marked dilation of the prostatic urethra without significant parenchymal effect. Three of the treatments (IC, ILC, TUMT) resulted in parenchymal ablation with only minor dilation of the urethra. Although each technique has encouraging experimental findings, ultimate success will be determined by further definition of the instrumentation technique and appropriate clinical implementation.
\end{abstract}

\section{INTRODUCTION}

Benign prostatic hyperplasia $(\mathrm{BPH})$ and prostate cancer are common features of male aging. BPH is considered to be the most common urinary problem in men over the age of

Keywords: Prostate, Hyperplasia, Cancer, Treatment, Hyperthermia, Laser, Stent, Experimental, Dog, Cryotherapy

\footnotetext{
"Correspondence: Email: jack.hoopes@dartmouth.edu; WWW: http://dartmouth.edu; Telephone: 603-650-5031 
50 years and prostate cancer is currently believed to be the most common male cancer. BPH and prostate cancer treatments are extremely important components of urologic practice, and result in about 2.5 million physician office visits and 600,000 prostatectomies/year in the United States. In spite of the high prevalence of BPH and prostate cancer and the large amount of medical resources devoted to treating them, there is comparatively little evidence regarding the etiology, progression and treatment of these diseases.'

Although prostate cancer is relatively uncommon in mammals other than man, prostate hyperplasia is very common in older dogs and, like men, the incidence increases with advancing years. Although the morphology of BPH is slightly different for dogs and men, the similarities are many and the canine prostate is an excellent model in which to study BPH and prostate cancer treatments. ${ }^{2}$

We have used the canine prostate model in all five of the experimental BPH/cancer treatment modalities presented herein. All studies in this series were performed at Dartmouth Medical School/Dartmouth-Hitchcock Medical Center, Lebanon, NH, and were supported in part by corporate and/or NIH funding. The study goal was to assess the feasibility and relative toxicity of each modality. Although the treatment results can not be specifically used to determine efficacy in human patients, there are a number of aspects of treatment pathophysiology and tissue response that should be favorably comparative between dog and man. Additionally, it is hoped that experimentation by a single investigative team using a consistent animal model will allow a more direct and useful comparison of potential treatment effects/toxicities.

\subsection{Pathophysiology and Treatment of BPH}

Morphological features of the origin and evolution of BPH at both a gross level and at a microscopic level have been previously described. BPH nodules originate in a region of the prostate (transition zone and periurethral gland region) located near the proximal urethra and comprising about $2 \%$ of the total mass and 5 to $10 \%$ of the mass of glandular tissue. Three pathological processes leading to benign prostate enlargement have been identified: diffuse cystic parenchymal enlargement, diffuse transitional zone enlargement, and nodule enlargement. ${ }^{3}$

An understanding of the mechanisms regulating prostate growth can be achieved only by full comprehension of the morphology of the gland. The prostate is a secretory organ arranged in an arborized array of ducts containing a number of different cell types. The proliferative glandular region at the tip of the duct, and the secretory region in the distal and intermediate segments, account for approximately $90 \%$ of the ductal system. The non-secretory cells located in the proximal region of the duct and immediately adjacent to the urethra, account for the final 10\%. Surrounding the ductal regions are the stromal fibromuscular components of the prostate, which support the epithelial outgrowth. On the basis of epithelial/mesenchymal (stromal) recombination studies, it now appears that during development, epithelial cells are directed by stromal signaling toward specific pathways of differential function. Although the process that induces ductal morphogenesis is androgen-sensitive, differences exist in the response to the androgens, depending on the region of the ductal system. ${ }^{4}$

The hormonal issues surrounding BPH physiology are not believed to be critical to the acute success or failure of the BPH gland to non-pharmacologic treatments. However, it 
is likely that long-term treatment response of a prostate to non-pharmacologic treatment depends, to some degree, on the healing reaction, which is influenced by sex hormone levels and physiology.

Surgical treatment of BPH via transurethral prostate resection is the second most common surgical operation performed in the U.S. and represents $38 \%$ of all major operations performed by urologists.

Although a number of techniques can be used to accomplish the BPH treatment procedure, the most commonly used procedure at this time is electrocautery resection through a resectoscope under general anesthesia. Major complications of this treatment (transurethral resection of the prostate or TURP) include bleeding, the need for catheterization (possibly up to 3 months post-surgery), long-term incontinence (1-3\%), and impotence $(4-40 \%)$; although this treatment is generally effective. ${ }^{6}$

\subsection{Pathophysiology and Treatment of Prostate Cancer}

Cancer of the prostate is the most common malignant transformation occurring in men, accounting for $20 \%$ of all male cancers. An estimated 244,000 new clinical cases were diagnosed in the United States in 1995 alone. Cancer of the prostate now causes $12 \%$ of all male, cancer deaths in the United States making it second to lung and equal to colorectal cancers as the most lethal cancer in man (American Cancer Society, 1992). The average man has a 30-40\% risk of developing carcinoma of the prostate: his risk of developing clinical prostate cancer is $10 \%$, and his risk of dying of prostate cancer is $3 \%$.

The aggressiveness of prostate cancer varies greatly. Many are extremely slow growing and prompt a laissez-faire diagnosis, with the assumption that the patient will succumb to other factors first. Indeed, autopsy studies have shown prostate cancer in as many as 40 percent of patients over the age of 75 years. However, even though many prostate cancers demonstrate an extremely long doubling time, the true malignant power of the cancer should not be under estimated; the cancer has a strong propensity to metastasize and to evolve into more aggressive forms. ${ }^{8}$

Prostate cancer originates in three glandular zones -- the peripheral, transitional, and central zones. Seventy to eighty percent of the prostate cancers originate in the peripheral zone, usually in close proximity to the prostate capsule. Another ten to twenty percent of the cancers originate in the transitional zone, with only five to ten percent originating in the central zone of the prostate. Thus, relative to its volume, the central zone is relatively resistant to the development of prostatic cancer. However, peripheral zone cancers can readily invade the central zone. ${ }^{8}$

Prostate cancer, like many other cancers, does not have precisely known and documented etiological factors. However, like BPH, age and sex hormone status are determinant factors. Although localized prostate cancer is most effectively treated by surgical prostatectomy and/or radiation, there are subsets of prostate cancer which could, theoretically, be effectively treated by more conservative modalities which have a reduced chance of significant complication. Additionally, some of these "less" invasive modalities might be extremely useful for patients who are poor surgical risks, or when minimally invasive palliation is important for mandatory life quality. 


\section{NON-SURGICAL, NON-PHARMACOLOGIC BPH / PROSTATE CANCER TREATMENTS}

All experiments were conducted in the Surgical Research Laboratory at Dartmouth Medical School (DMS). All animal experimentation at DMS was reviewed and approved by the Dartmouth College Institutional Animal Care and Use Committee, and was overseen by the Dartmouth College Animal Care and Use Program which is certified by the American Association for Accreditation of Laboratory Animal Care and operates under USDA and PHS guidelines.

The five non-surgical, non-pharmacologic experimental BPH and/or prostate cancer treatment modalities evaluated in this review include: (1) transurethral, radiofrequency heated water-filled (thermal) balloon; (2) transurethral, water-cooled microwave hyperthermia; (3) transurethral/transrectal, air-cooled hyperthermia; 4) interstitial laser coagulation; and (5) interstitial cryotherapy. Each modality is discussed in terms of historical use and its experimental clinical and clinicopathologic findings in the canine model.

General experimental methods include anesthesia pretreatment with atropine sulfate $(0.02$ $\mathrm{mg} / \mathrm{kg}$ ), followed by injectible intravenous induction anesthesia, endotracheal intubation and anesthesia maintenance with a $0.5 \%$ to $5.0 \%$ halothane/isoflurane mixture via a semiclosed circuit.

In treatment preparation, all dogs were placed in dorsal recumbancy and their abdomens and perineums prepared and draped for standard, aseptic surgery. In some dogs a perineal urethrostomy was performed in order to place a treatment device into the prostatic urethra in retrograde fashion. However, high post-surgical complication rates led us to place most transurethral applicators into the prostatic urethra in an antegrade fashion through a cystotomy incision. Following routine surgical closures, many but not all, dogs were catheterized for 2-5 days.

Normal body temperature was maintained throughout the experimental treatment. Warm saline was poured into the abdominal cavity to minimize the cooling that took place from the exposed viscera.

Blood samples were obtained by cephalic vein puncture. Urine samples were obtained free-catch during a normal urination procedure. Analysis of hematological and urinalysis parameters was performed at Dartmouth-Hitchcock Medical Center.

Dogs were euthanized with intravenous saturated $\mathrm{KCl}$ during surgical plane anesthesia (induced as described above). Following euthanasia, the prostate was measured and the gross description recorded. The prostate, rectum and membraneous urethra were removed en block and emersion-fixed in $10 \%$ neutral-buffered formaldehyde.

Following fixation, the prostates were trimmed in longitudinal and/or transverse (axial) plane and cut to correspond to imaging planes (if appropriate). This technique allowed accurate co-registration of the imaging and histopathologic studies. In order to quantify the volume of each tissue type that composes the prostates, a grid-based histomorphometric analysis was performed using hematoxylin/eosin, Masson's Trichrome, and/or PAS stained slides of each prostate studied. The prostate tissue type/category under each grid cross-hair was evaluated and placed in one of 4 groups (normal glandular tissue; inflamed glandular tissue; stroma; lumen space). Some types of pathologic effects (area, 
percent component assessment/comparison of remaining tissue, fibrosis, cavitation, necrosis and/or inflammation) and were also quantified by automated image analysis using the microscope, a video camera, and a Macintosh with NIH Image ${ }^{\circledR}$ software. These techniques allowed accurate quantitation of tissue change following treatment as well as meaningful co-registration of treatment and imaging parameters with pathology.

In order to have a histologic baseline for comparison, two normal canine prostates were histologically quantitated in a manner identical to that used on the treated glands.

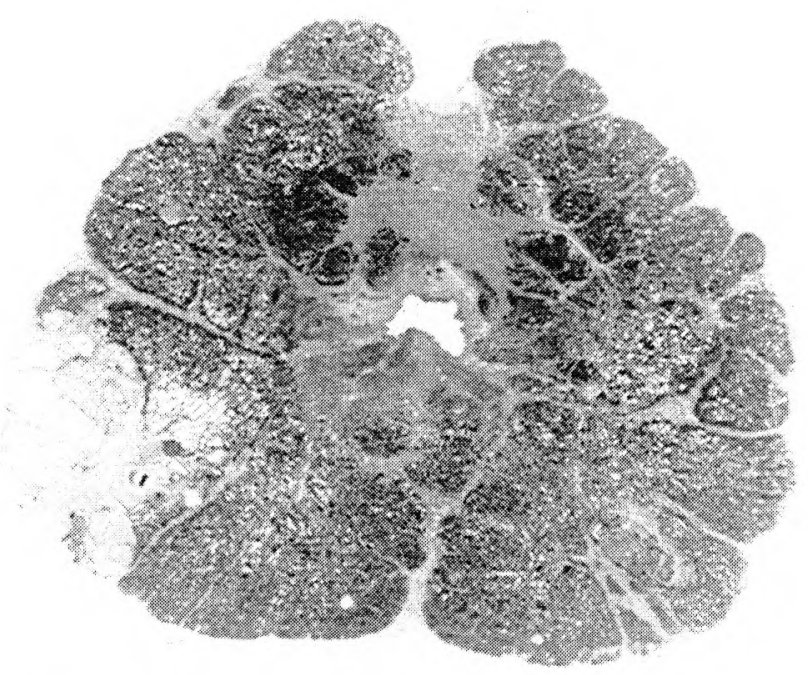

Figure 1. Full-face histological section of a BPH canine prostate showing moderate uniform hyperplasia with pronounced cystic alteration in the lower-left corner.

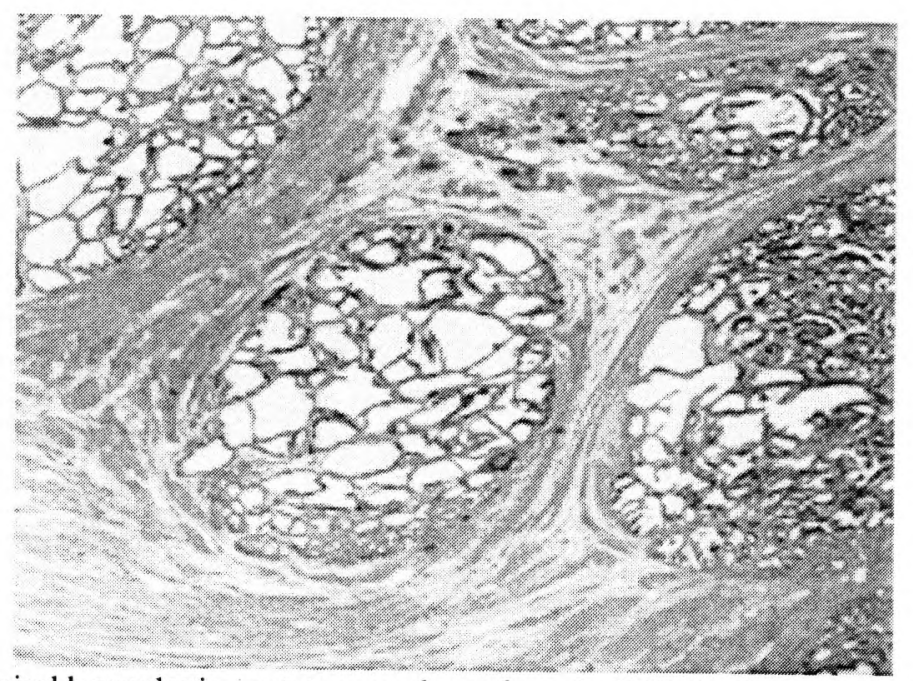

Figure 2. Typical hyperplastic prostate parenchyma from the BPH gland seen in Figure 1; focal, glandular hyperplasia is seen between thickened bands of stroma. (H\&E; 25x mag.) 


\subsection{Transurethral Radiofrequency Thermotherapy (TURT)}

Radiofrequency has been used in a variety of methods to treat BPH-induced urinary obstruction. These methods primarily include techniques aimed at using a radiofrequency antenna to directly ablate prostate parenchyma. In general, these techniques have been reasonably successful in relieving acute obstruction symptoms with moderate retention rates $(20-30 \%){ }^{9}$

In a recent limited clinical trial, Dawkins, et al., ${ }^{10}$ showed radiofrequency-induced intraurethral hyperthermia $\left(48^{\circ} \mathrm{C} / 3 \mathrm{hr}\right)$ which resulted in a $70 \%$ favorable outcome in symptomatic urinary obstruction scores. This favorable result decreased to $40 \%$ by 3 years, and to $22 \%$ by 4 years. A second recent study $y^{11}$ evaluated 49 patients 3 months following TURT. Only $14 \%$ of these patients achieved excellent/good results. Complications included gross hematuria and erosion of the external meatus in 5 patients; however, none of the patients had urinary retention.

Heated water-filled balloon dilatation is a potential single-treatment minimally-invasive alternative to TURP. With direct placement in the prostatic urethra, the thermal balloon should effectively transfer cytotoxic heat to the prostatic tissue immediately surrounding the urethra, inducing cell death and increasing urethral diameter, thereby relieving the obstructive symptoms of BPH.

A two-dog pilot study was designed to determine if thermal balloon dilation technology was capable of providing safe chronic dilatation of the prostatic urethra. The treatment goal was to increase urethral diameter and patency through urethral mucosa and periurethral cell death without extending damage longitudinally to the sphincters or radially beyond the prostate parenchyma/capsule.

\subsubsection{Methods of TURT}

Standard anesthesia and surgical preparation was used. A contrast urethrogram was performed to document the patency of the prostatic urethra prior to treatment. Following the contrast urethrogram, a deflated-balloon catheter was placed in the prostatic urethra. A laparotomy was performed so that the thermistors could be accurately placed and the prostate visualized during the treatment. The rectal thermistor was placed along the dorsal rectal mucosa as closely aligned to the center of the prostate as possible. The distance from the center of the prostatic urethral catheter to the rectal mucosa and thermistor was approximately $20 \mathrm{~mm}$. The dorsal capsule of the prostate and the rectal serosa were in immediate apposition.

A thermal prostate balloon $(10 \mathrm{~mm} \times 2 \mathrm{~cm})$ was placed in the prostatic urethra between the internal and external sphincter. The balloon was heated to $50-55^{\circ} \mathrm{C}$ (measured at a single, internal thermistor located approximately midway between the two, RF electrode rings) for 15 minutes at 2 ATM. The external sphincter was actively cooled via a controlled room-temperature saline flush.

In order to understand and document the urethral and rectal temperature profiles, thermometry pullbacks were performed at $2.0 \mathrm{~mm}$ increments using a Luxtron 3000 fiberoptic probe within a catheter along the outer length of the balloon. 
Matching the Energy Source to the Clinical Need / 525

\subsubsection{Treatment and clinical outcome of TURT}

Rectal temperatures ranged between $38.0-38.8^{\circ} \mathrm{C}$ in both dogs. Urethral heating peaks were seen between the proximal RF electrode and the proximal edge of the balloon. The maximum measured balloon urethral-temperatures were $61^{\circ} \mathrm{C}($ Dog 1$)$ and $53.2^{\circ} \mathrm{C}($ Dog 2), as shown in Figure 3.

Clinically, both dogs survived the 5-day treatment without complication and at the time of sacrifice, were continent. Urethral catheters were removed at 18-24 hours posttreatment. Although there was dysuria, the dogs continued to urinate throughout the study.

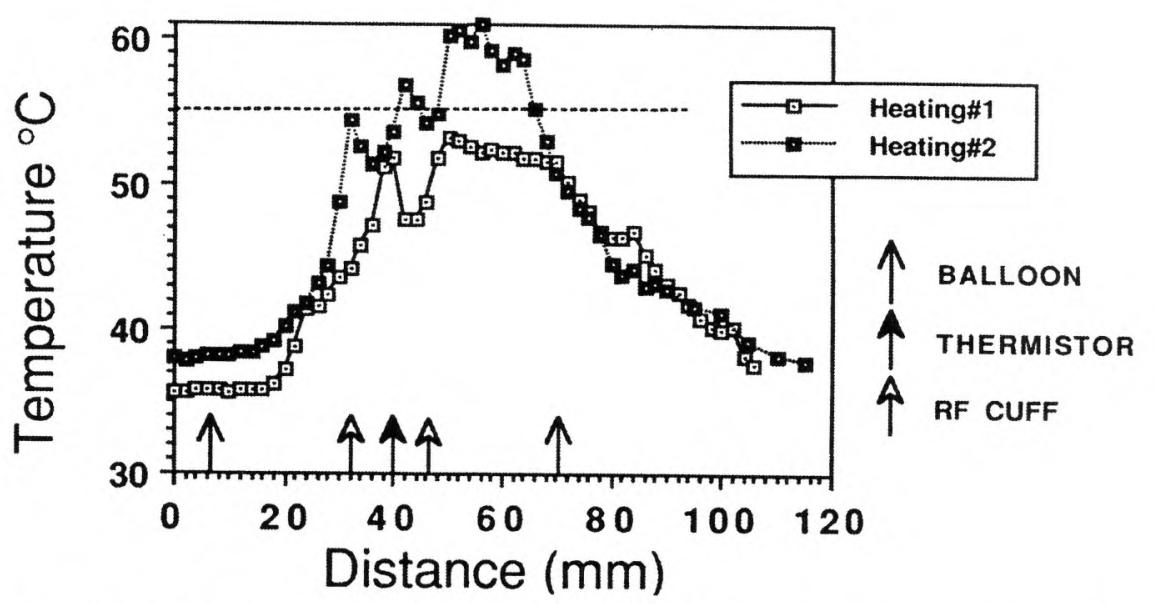

Figure 3. These curves represent temperature profiles of the prostatic urethra during the 15 minute heat period. The temperature probe was located inside a catheter that was situated between the balloon and prostatic urethra. The arrows depict the relative position of the temperatures to the RF probe and the balloon that spared the entire prostatic urethra.

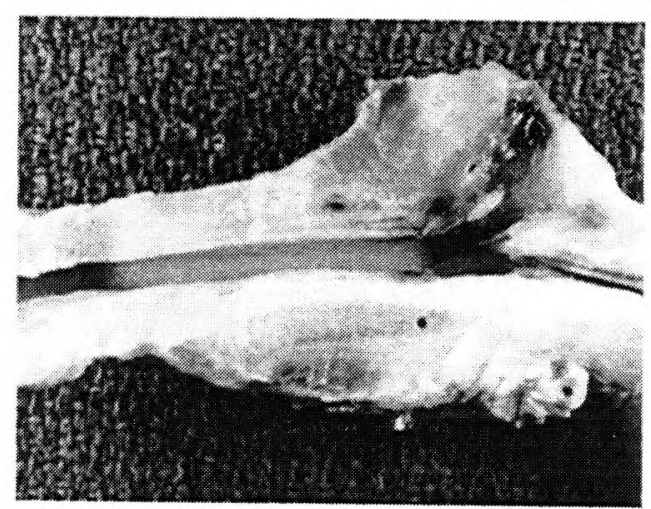

Figure 4, Longitudinal, mid-sagittal plane of prostate (Dog \#2), $48 \mathrm{hr}$ post-treatment. Note urethral and parenchymal hemorrhage in the region of the urethral sphincter and bladder neck. The posterior potion of the prostatic urethra is unremarkable. 
Urinalyses were performed before treatment; in the acute post-treatment time; posttreatment days 1-5; and at the time of sacrifice (post-treatment day 14). Moderate hematuria and proteinuria were seen throughout the post-operative period with the greatest increase in inflammatory and epithelial cells seen at post-operative days 3-5.

\subsubsection{Gross and histopathology results of TURT}

At the end of day 5 of the follow-up period, the prostates showed focal hemorrhage in the anterior parenchymal and sphincter regions. The urethras were mildly dilated and had marked mucosal denudation/ulceration.

The most significant pathologic changes included scattered hemorrhage, inflammation and ulceration primarily confined to the anterior prostate and bladder neck/internal sphincter region. The middle and posterior urethra were generally unremarkable, except for scattered inflammation. The submucosa contained regions of hemorrhage (petechia), mild edema and inflammation. Most of the submucosal inflammation in the periurethral region was lymphoplasmacytic with a scattering of neutrophils. There is moderate gland atrophy with accompanying fibrosis in the transitional submucosal region. Selected regions of the glandular parenchyma (generally deep in the gland) contained large pockets of neutrophils.

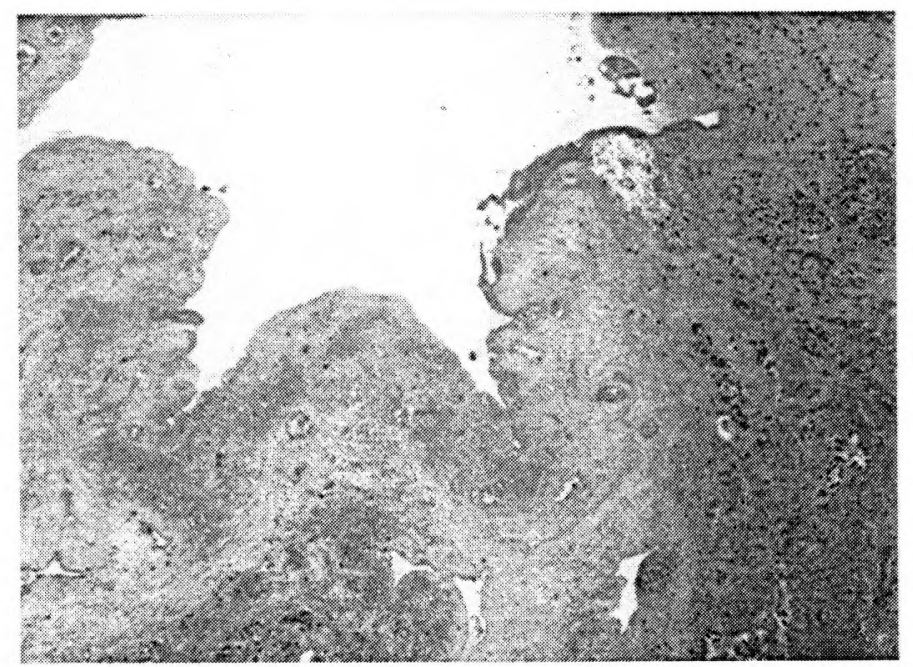

Figure 5. Anterior prostatic urethra 5 days following a $15 \mathrm{~min}, 54^{\circ} \mathrm{C}$, thermal balloon dilation. Note complete loss of mucosa/epithelium and denaturation of the submucosal parenchyma and connective tissue. (H\&E; 25x mag.)

\subsubsection{Conclusions of the TURT modality}

It is not precisely known why the greatest treatment effect was seen at the anterior/sphincter region; however, it may have been due to the tight proximity of this tissue to the balloon, or the slightly off-centered (anterior) location of the hottest portion of the antenna (although the fluoroscopy studies prior to the treatment indicated this was not the case). 
Overall, this therapy (at the thermal dose used) appears to deliver a relatively shallow cytotoxic effect to the mucosal and submucosal tissues. It is a virtual certainty that higher temperatures and/or longer heat times would increase the cytotoxicity in the periurethral tissues and the magnitude of the urethral dilitation. Although the convective heating nature of the treatments suggest that it would be safe for tissues not in close proximity to the applicator, the high urethral-temperatures could prove troublesome in the acute posttreatment time-period, and to the spincters and bladder neck.

\subsection{Transurethral, Microwave Thermotherapy (TUMT)}

Microwave hyperthermia has been evaluated experimentally and clinically as a nonsurgical treatment for BPH and prostate cancer. Preliminary reports using both transurethral and transrectal applicators have demonstrated varying subjective and objective response rates. One of the issues that has not been addressed adequately is predicting, a priori, which group of patients will benefit from this modality. Prostate tumors and BPH are relatively vascular-deficient and therefore poorly equipped to dissipate the destructive effects of hyperthermia. Therefore, these conditions should "theoretically" be sensitive to hyperthermia. The chief concerns for using hyperthermia to treat BPH and prostate cancer are similar: the inability to direct the heat appropriately in the parenchyma while simultaneously sparing the urethral mucosa, sphincters and the extra prostate tissues such as rectum.

Two recent manuscripts ${ }^{12.13}$ have shown that factors such as prostate vessel density and epithelium/stroma $(\mathrm{E} / \mathrm{S})$ ratio, location of the heat maximum as well as prostate size, age and symptom score were important factors in the ultimate success of TUMT. This new information shows that patients who respond poorly to TUMT had high vessel densities and lower E/S ratios. Long-standing information shows that increasing patient age, prostate volume and decreased urinary flow rate are negative predictors for TUMT success. $^{14}$

The purpose of this study was to determine the acute and chronic effect of a $48^{\circ} \mathrm{C} / 60 \mathrm{~min}$ transurethral microwave $(915 \mathrm{MHz})$ hyperthermia treatment in the canine prostate.

\subsubsection{Methods of TUMT}

The prostates of 14 dogs were treated using water-cooled transurethral microwave hyperthermia for 60 minutes. Treatment guidelines included a $40^{\circ} \mathrm{C}$ maximum in the urethra, $42.5^{\circ} \mathrm{C}$ maximum in the rectum and a target temperature of $48^{\circ} \mathrm{C}$ or greater in the prostate. Treated prostates were examined by urinalysis, transrectal ultrasound (TRUS), and histology, between 7 and 120 days post-treatment. The transurethral treatment catheter contained a choked dipole micowave antenna (operating at $915 \mathrm{MHz}$ ) which was surrounded by circulating $20^{\circ} \mathrm{C}$ deionized water.

Following standard anesthesia and surgical preparation, perineal urethrostomy and laparatomy procedures were performed to place a retrograde balloon treatment catheter into the prostatic urethra with the non-heating tip extending into the bladder. Target balloon dilatation was $2.0 \mathrm{~cm}$ at a pressure of $14 \mathrm{psi}$. The junction of the antenna was centered in the prostate. Five, 18 gauge catheters (providing tracks for fiberoptic probes) were inserted into the prostate to monitor (map) temperatures. The thermometry catheters were placed parallel (anteroposterior) and perpendicular (dorsoventral/mediolateral) to the antenna. Automated temperature measurements, using Luxtron fiberoptic probes, 
were made every five minutes at $1.0-2.0 \mathrm{~mm}$ intervals along the entire length of the thermometry catheters. Temperatures were also monitored in the rectum, along the urethra proximal to the antenna choke, and in the urethra adjacent to the antenna junction. Power was adjusted manually to obtain $48^{\circ} \mathrm{C}$ or greater in the prostate. The prostate margins relative to each catheter were carefully documented and monitored so that intraand extra-prostate comparisons of the heat treatment could be made. A period of approximately $10 \mathrm{~min}$ for initial heat-up preceded the $60 \mathrm{~min}$ treatment at steady state. Typically between $40-50 \mathrm{~W}$ of power was required to achieve the prescribed thermal dose.

\subsubsection{Treatment and clinical outcomes of TUMT}

Although many dogs were catheterized for 3-5 days, even those who removed their own catheters as-soon-as $24 \mathrm{hr}$ post-treatment, had minimal difficulty urinating. Serum biochemical (acid phosphatase, alkaline phosphatase, BUN, creatinine) and standard urinalysis assessment showed mild to moderate increase in the aforementioned assays as well as increases in RBC, WBC and epithelial cells in the urine. All of the above increases reached their highest levels between 5-14 days and were within normal range by 30 days post-treatment. TRUS assessment of the treated prostates at 14,30 and 120 days post-treatment showed variable hyperechoic and hypoechoic zones in the central and outer prostate in most dogs. By 30 days post-treatment, the hyperechoic zones (which correspond primarily with inflammation and gland atrophy) were less pronounced sonographically. The hypoechoic zones (corresponding with cavitation/necrosis) were present at all time-periods. The size of the cavitated region increased in conjunction with thermal dose. However, at 120 days post-treatment some cavities were sonographically smaller. This change appears to be due to overall prostate shrinkage and possibly fibrotic contraction around the cavities.

\subsubsection{Gross and histopathology results of TUMT}

All prostates treated with transurethral microwave hyperthermia demonstrated similar types of gross change including cavitation and acute, mild urethral-dilatation.

Parenchymal cavitation was observed as early as the 14-day post-treatment end-point,

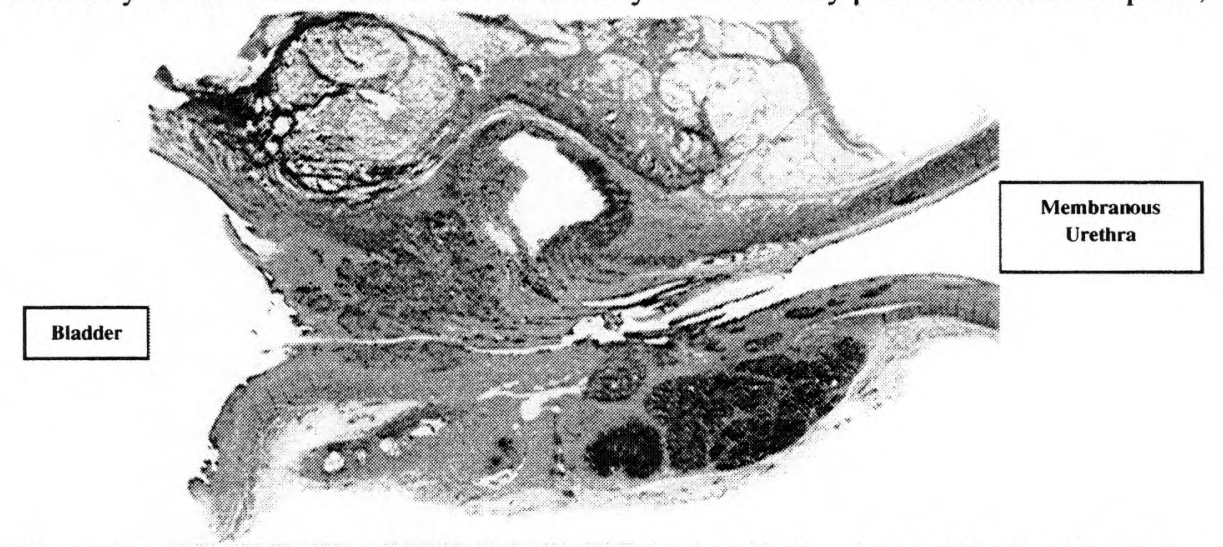

Figure 6. Full-face sagittal-plane histological section of a canine prostate 30 days following cooled transurethral microwave hyperthermia. A maximum thermal dose of $48-50^{\circ} \mathrm{C} / 60$ min was achieved in the cavitated region in the center of the ventral lobe. The dog was urinating normally and the urethra and periurethral tissues were unremarkable at the time of sacrifice. (H\&E) 
and persisted throughout the 120-day study, although the amount of cavitation appeared to decrease slightly at the latest assessment period.

Histologic assessment of hyperthermia-treated prostate glands showed various levels of focal necrosis/cavitation, fibrosis, inflammation, and glandular atrophy. The cavitation was often confined to a focal region representing approximately $20-25 \%$ of the prostate tissue. Depending on the time post-treatment, regions of surviving and/or regenerated glands were seen between pie-shaped regions of damage or inflammation that apparently correspond to the insular supply. Increased fibrosis was often, but not always, seen in the region of parenchymal loss and in areas normally occupied by stroma. Aseptic chronic or active inflammation of various intensities was present at all assessment periods, but was markedly decreased at the later times post-treatment and was asymmetrically distributed primarily in the middle and outer regions of the gland. The urethra was not histologically damaged in any of the dogs, and the periurethral tissue ( $5 \mathrm{~mm}$ from the urethral wall) was also largely free of damage, as were sphincter regions and the bladder neck.

\subsubsection{Conclusions of the TUMT modality}

Cooled, transurethral microwave hyperthermia of the prostate achieving temperatures of $48^{\circ} \mathrm{C}$ or higher for 60 minutes resulted in focal necrosis (cystic cavitation) marked focal inflammation, parenchymal atrophy, stromal proliferation and replacement fibroplasia. Although the highest thermal doses resulted in cavitation, slightly-lower doses $\left(44^{\circ} \mathrm{C} / 60\right.$ $\mathrm{min}$ ) resulted in significant parenchymal atrophy and fibrosis. Correlation of the pathologic effects and temperature maps showed, as expected, increased damage in the regions that received the highest thermal doses. Histomorphometry assessment of the treated prostates demonstrated a marked increase in the volume of fibromuscular stroma (as compared to glandular parenchyma) 30 days post-treatment. The difference remained significant, but was less marked, at the latest (120-day) end-point. Focal fibrosis of the dorsal prostate capsule and adjacent rectal serosa occurred in two dogs whose prostate diameters measured $<3 \mathrm{~cm}$. These small prostates, in which the target tissue was the same distance from the applicator, received higher thermal doses at the capsule (range 42.5-43.

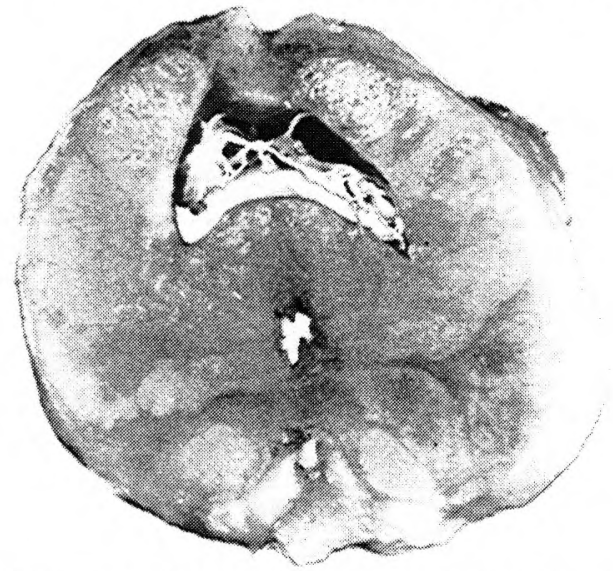

Figure 7. Full-face axial/transverse plane section of a canine prostate 120 days following cooled transurethral microwave hyperthermia. A maximum thermal dose of $48-50^{\circ} \mathrm{C}$ was achieved in the cavitated region in the central, dorsal prostate. Mild urethral dilitation and scattered (but often significantly large) $\mathrm{T}$-cell infiltrates were also seen in the treated prostates. 


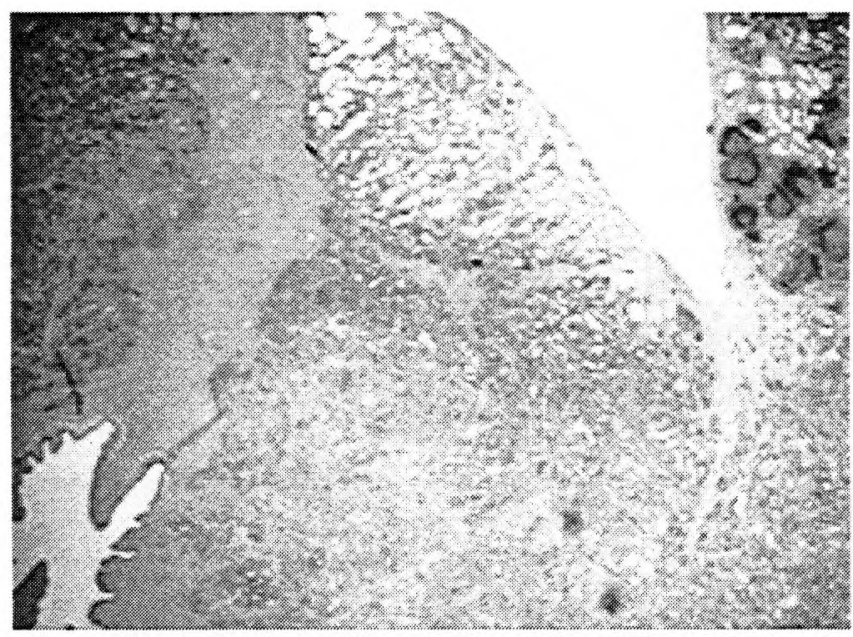

Figure 8. Photomicrograph of a portion of the prostate section of Figure 7, showing relatively normal/hyperplastic prostate parenchyma between the urothelial-lined cavitation (upper right) and the urethra (lower left). This, and similar sections, suggested that the urethraV/antenna-cooling mechanism effectively protected the urethra and periurethral tissues from chronic heat damage; and that the target thermal dose $48^{\circ} \mathrm{C} / 60 \mathrm{~min}$ achieved the desired parenchymal ablation; albeit to a lesser volume than might be desired or anticipated. (H\&E; 10x mag.)

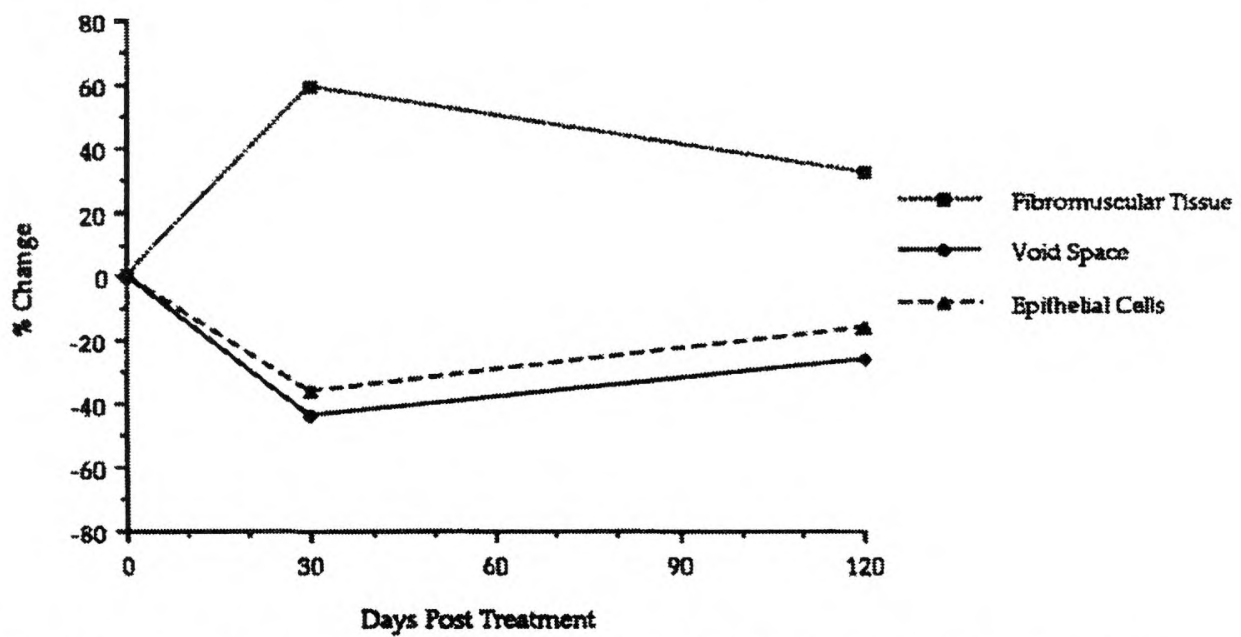

Figure 9. This graphic demonstrates the relative percent change in prostate tissue component volume following transurethral hyperthermia $(\mathrm{N}=4 \mathrm{dogs})$. There was a marked increase in fibromuscular and FCT stroma and a corresponding decrease in glandular volume (epithelium and lumen or cystic/cavitation space). The increase in fibromuscular/FCT stroma was slightly greater at 30 days than at 120 days. This reaction was likely due to the relatively larger amounts of injured parenchyma and inflammation seen at 30 days. The relative decrease in damaged tissue and inflammation, and overall shrinkage/consolidation of the gland, accounted for changes in slope direction between 30 and 120 days. 


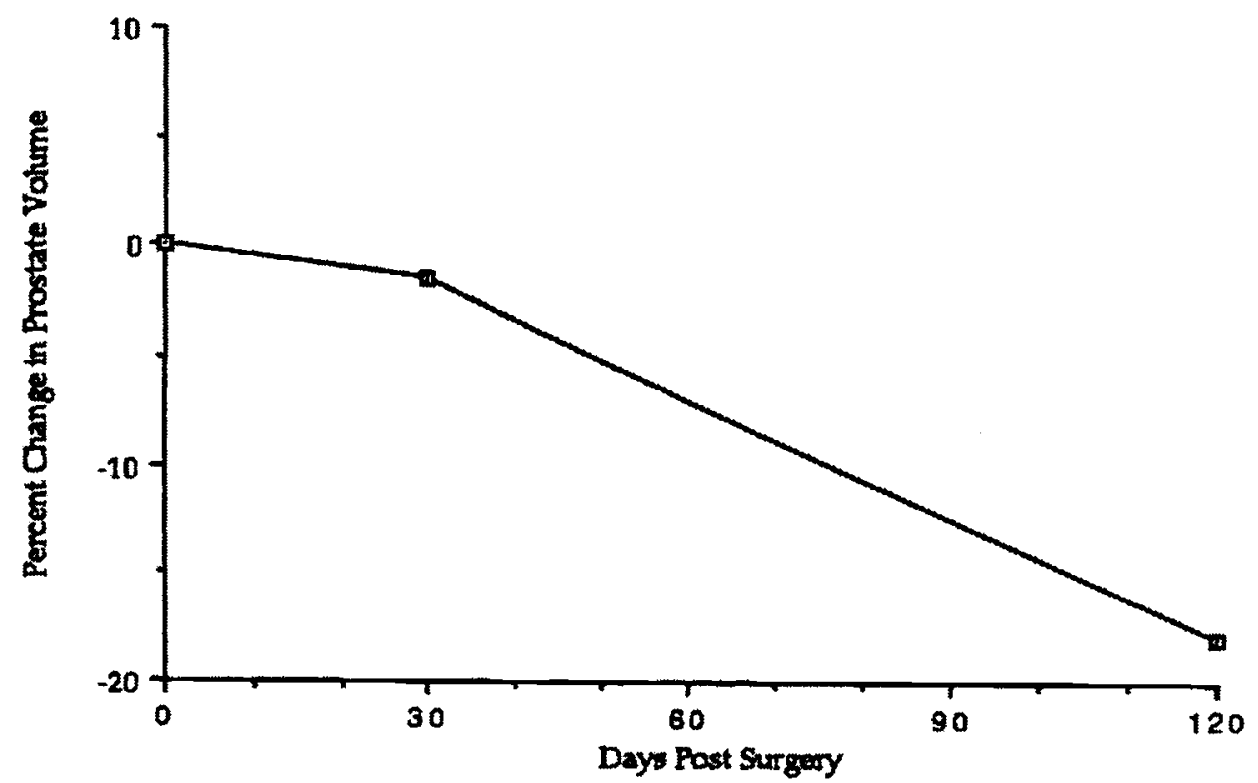

Figure 10. This graphic demonstrates the relative mean percent change in prostate size following treatment by transurethral microwave hyperthermia $(\mathrm{N}=4 \mathrm{dogs})$. The total volume of glandular tissue was reduced by nearly $20 \%$ at 120 days post-treatment. Prostate shrinkage appeared to continue as the cavitation and damage were replaced by FCT scar.

$43.5^{\circ} \mathrm{C}$ ). In the larger prostates, the periprostatic temperatures were lower (range 41 $\left.42^{\circ} \mathrm{C}\right)$.

\subsection{Transurethral/Transrectal, Microwave Thermotherapy (TUMT/TRMT)}

Transurethral/transrectal microwave hyperthermia is conceptually similar to that discussed in the previous transurethral hyperthermia (TUMT) section. Although there is little published information relating to the use of simultaneous transurethral and transrectal hyperthermia for prostate disease, there is abundant information on the use of transurethral microwave hyperthermia alone and limited reporting of transrectally delivered microwave hyperthermia of the prostate. Barbalias, et al., ${ }^{14}$ showed that transrectal microwave thermotherapy (TRMT) with urethral cooling at 5 or 6,1 hour sessions $\left(42-43.5^{\circ} \mathrm{C}\right)$ alleviated $75 \%$ of obstructive symptoms, including increased maximal-flow rates and decreased residual urine levels. In a similar study, Stawarz, et al., ${ }^{15}$ showed that TRMT, 6 sessions $\left(43.5^{\circ} \mathrm{C} / 30 \mathrm{~min}\right)$ resulted in improvements in immune $(\mathrm{T}$-cell) function in prostate cancer patients. The improvements peaked at 2 months and decreased to pre-treatment levels by 6 months post-treatment.

The object of our study was to develop a dual-antenna phase-control hyperthermiasystem capable of delivering a controlled thermal-dose to the prostate while sparing sensitive rectal tissue. Such a system should be useful in treating selective BPH and prostate cancer patients who are poor candidates for surgery and/or whose lesions are anatomically appropriate (dorsal and dorso-lateral prostate lobes) for this treatment modality. 


\subsubsection{Methods of TUMT/TRMT}

Using our standard anesthesia and surgical (laparotomy) protocols, 8 dogs were treated with the transurethral and transrectal prostate hyperthermia applicators. Four dogs were sacrificed acutely, and 4 dogs were followed for 30 or 60 days.

Two microwave applicators capable of using phase control were developed to produce controlled elevation of temperature in the prostate. A flexible $6 \mathrm{~mm}$ applicator was designed for the urethra. This applicator incorporated a choked, resonant microwave dipole with an omni-directional heating pattern and an air-cooling system to control the temperature of the urothelium. A second $18 \mathrm{~mm}$ rigid applicator was designed for use in the rectum. It incorporated an eccentric, choked, resonant microwave dipole that radiated toward the prostate with a front-to-back power ration of 20 . An air-cooling system controlled the temperature of the rectal mucosa. The applicators were driven at $915 \mathrm{MHz}$. The use of phase control will theoretically allow the production of directed thermaldoses, high enough to create controlled tissue ablation in the regions of the prostate, which are accessible by energy from both antennas.

The objectives of the hyperthermia treatment were: (a) $\geq 43^{\circ} \mathrm{C}$ in the prostate parenchyma; (b) $\leq 40^{\circ} \mathrm{C}$ in the rectal serosa; and (c) $\leq 41^{\circ} \mathrm{C}$ in the urothelium for a single 30 minute treatment. Following exposure of the prostate, the applicators were aligned in the central prostate region. Fiber optic probes were placed within the catheters implanted in the prostatic parenchyma, pre-prostatic tissue, rectum, and the outer surfaces of the applicators. Low-power heating was then used to provide optimal positioning.

The applicator surfaces were cooled by adjustable room-temperature air-flow. Thermometry catheters were placed on the anterior, lateral, and posterior quadrants of the applicators for measurement of temperature elevation in the rectum and urethra.

Microwave power was slowly adjusted upwards over a 10-minute heat-up period to achieve optimal power levels. Temperature mapping was performed at 10 -minute intervals.

\subsubsection{Treatment and clinical outcome of TUMT/TRMT}

Complete blood counts (CBC); serum biochemical indicators of prostate damage (acid phosphatase, alkaline phosphatase); urinary obstruction (creatinine, BUN); and standard urinalysis examinations were mild to moderately elevated in the initial post-treatment time, but had essentially returned to normal by post-treatment day 14 .

Indwelling urinary catheters were not placed post-operatively. All dogs had mild dysuria over the initial 2-3 days, but had recovered completely normal function by 7 days posttreatment.

The chokes in the microwave applicators developed for this study restricted the axial heating distribution in vivo, thereby protecting the tissue overlying the feedlines. The aircooling systems limited the rectal, serosal and urothelial temperatures to about $40^{\circ} \mathrm{C}$, while permitting a maximum temperature of about $43( \pm 0.5)^{\circ} \mathrm{C}$ in the prostate. Rectal serosal temperatures limited the amount of power that could be applied safely. 


\subsubsection{Gross and histopathology results of TUMT/TRMT}

By 30 days post-treatment, the urothelium had fully regenerated, and the urethral lumens were mildly dilated. In one dog, a small region of rectal mucosa was eroded, and replaced with fibrous connective tissue. The greatest lumen area $\left(52 \mathrm{~mm}^{2}\right)$ was observed in the dog that received the greater thermal dose $(50 \mathrm{~min})$. The smallest area $\left(3 \mathrm{~mm}^{2}\right)$ was observed in the dog sacrificed after the shortest follow-up period, 29 days. For comparison, the three acute dogs had an average urethral cross-sectional area immediately after treatment of $2.2( \pm 1.5) \mathrm{mm}^{2}$ (as compared to no lumen space in the normal dog).

Focal lymphocytic inflammation of the prostate, and periurethral fibrosis were observed in all dogs (to varying degrees).

\subsubsection{Conclusions of the TUMT/TRMT modality}

In spite of the lack of parenchymal damage (between the two antennae) in the dorsal prostate lobe, the choice of a 270-degree phase delay for the urethral applicator appeared to be appropriate. Our preliminary measurements of point temperatures in both tissue phantom and the acute subjects with various phase shifts confirmed that rectal heating was minimized and central prostatic heating was maximized with this phase shift.

The directionality of the eccentric antenna within the rectal applicator was sufficient to protect the dorsal rectum from elevated temperature in all dogs except one (and that tissue was minimally over-heated). In vivo measurements showed that the dorsal rectal applicator surface was $4.5^{\circ} \mathrm{C}$ cooler than the ventral surface. That, in turn, was $3.5^{\circ} \mathrm{C}$

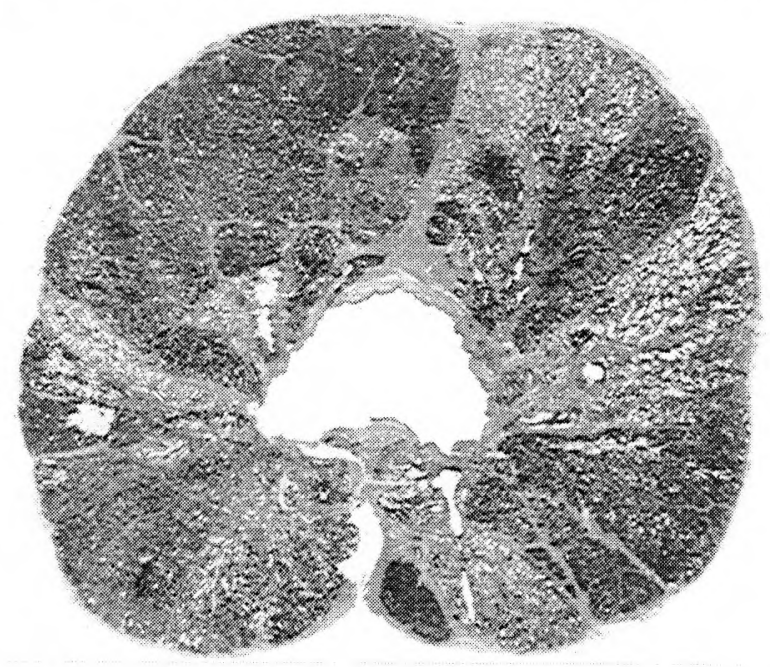

Figure 11. Transverse/axial section of a canine prostate 60 days post-treatment with transurethral, transrectal thermotherapy. Although the urethra was maintained at a thermal dose of $\leq 41^{\circ} \mathrm{C} / 30 \mathrm{~min}$, which would generally be considered non-damaging, the urethra was permanently dilated to a significant degree. Although it appears that the transurethral and transrectal antennas were too close (in proximity) to achieve optimal phase control and tissue effect between them, it may be possible that microwave energy from the transrectal antenna enhanced the transurethral energy, and subsequently the urethral dilation effect seen in both chronic dogs. 


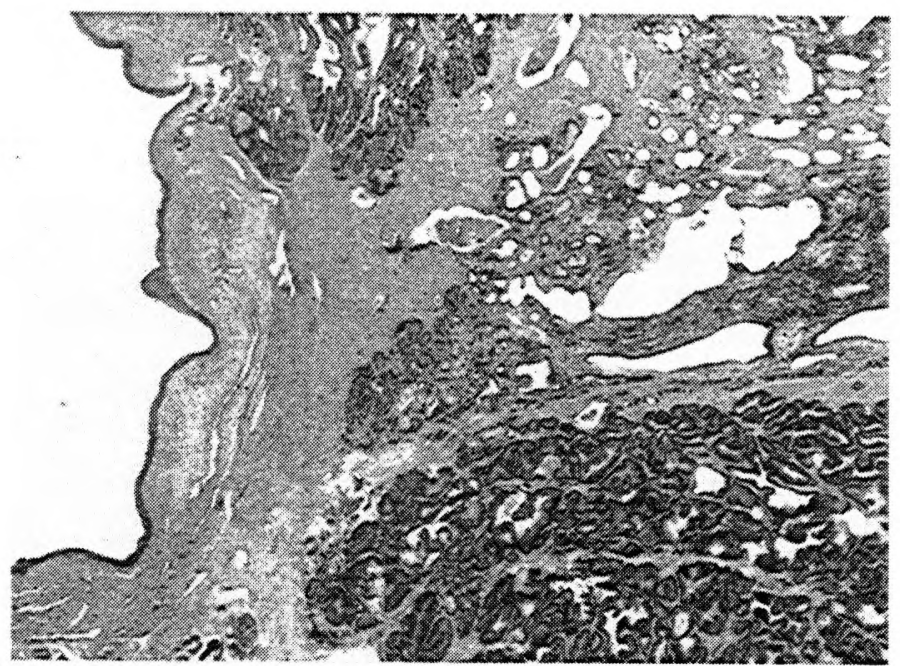

Figure 12. Photomicrograph of a portion of the dilated urethra (left) and adjacent parenchymal tissue from the prostate seen above (Figure 11). The urethra is completely lined by intact urothelium and, except for minor focal gland atrophy, the periurethral tissues are unremarkable. Similar urethral dilatation was seen in a second prostate that received an identical treatment. (H\&E; 10x mag.)

below the maximum allowed value of $40^{\circ} \mathrm{C}$. The values of air-cooling power that we observed ( 7 to 8 watts in both applicators) were significant fractions of the radiated microwave power (an indication that the surface cooling significantly modified the temperature distribution). We observed that the maximum urothelium temperature was $3.5^{\circ} \mathrm{C}$ below the peak temperature at a greater radius and at the same axial location. We observed a significant temperature gradient across the ventral rectal wall; the typical ventral serosal temperature was about $40^{\circ} \mathrm{C}$, while the ventral mucosal temperature was about $36.5^{\circ} \mathrm{C}$.

A trend between the thermal dose and the cross-sectional area of the urethra at two months post-treatment, was noted. All 60-day post-treatment dogs had significantly greater cross-sectional urethral areas than did the treated prostates measured immediately after treatment. The prostate assessed 30 days post-treatment had a urethral diameter similar to the acute dog.

Further studies are required to define why the phase-shift failed to create significant lesions in the parenchyma between the rectum and the urethra, yet did create larger urethral diameter than would have been expected by the thermal doses delivered by the urethral antenna alone. In comparison to the TUMT procedure, TUMT/TRMT did not require catheterization, and only mild dysuria was observed following the treatment. Room-temperature air proved to be an adequate coolant.

\subsection{Interstitial Laser Coagulation (ILC)}

Interstitial laser coagulation (ILC) is the interstitial application of laser energy to achieve tissue coagulation by a thermal process and is acheived by implanting the laser delivery fiber directly into the tissue. The advantage of the interstitial laser technique over photoirradiation of tissue by contact/non-contact lasers is the use of relatively low-power 
Matching the Energy Source to the Clinical Need / 535

laser light (2-10 watts) to cause coagulative necrosis of tissue. In addition to the lower energy requirements, ILC should allow the preservation of non-target adjacent tissues with appropriate monitoring.

ILC uses low powers but provides sufficient laser energy to heat cells throughout the target volume to temperatures exceeding protein denaturation thresholds, typically $60^{\circ} \mathrm{C}$ which induces coagulation necrosis using an $\mathrm{Nd}$ :YAG laser. Temperatures close to the implanted fiber may reach $100^{\circ} \mathrm{C}$.

Initial, experimental canine studies showed that large or small volume ILC lesions healed uneventfully following fibrocystic degeneration. Using $150-400 \mathrm{~mm}$ fibers and Nd:YAG laser treatment of 200-1500 sec (1-5 watts), McNicholas, et al., ${ }^{17}$ created well defined coagulation necrosis in the prostates of beagles. There was minimal charring or fiber damage and a dose of 1000 joules resulted in a $1 \mathrm{~cm}^{3}$ lesion. Tissue necrosis was negligible at doses below $650-700 \mathrm{~J}$.

Littrup, et al., ${ }^{18}$ using treatments of 15-60 watts for 5 seconds, have described changes similar to those shown by McNicholas.

A recent review ${ }^{19}$ of interstitial laser prostatectomy results suggests that this procedure can safely and effectively decrease prostate volume and increase urinary flow-rate. Since this treatment was not associated with significant blood loss or increased fluid shifts, it can be performed in higher-risk patients. Complications included retrograde ejaculation and uncomplicated urinary-tract infections are common but not serious or long-standing.

Our studies were designed to determine the use, feasibility and toxicity of ultrasoundguided interstitial laser coagulation in the canine prostate. Our treatment objectives were: (a) the transcapsular delivery of a 5 min interstitial laser-treatment in the prostate parenchyma using various power settings from 2-8 watts; and (b) the assessment of the treatment effects at various times from 0-60 days post-treatment, using TRUS and MRI.

\subsubsection{Methods of ILC}

Following standard anesthesia and surgical laparotomy, the laser fiber was delivered interstitially under TRUS guidance to the central region of each prostate lobe. Temperatures were recorded through 7 implanted metal-thermocouples ( 21 sensors) and a linear array of 10 thermal-sensors that were passed (in a catheter) via the bladder through the prostatic urethra. The interstitial thermocouples were implanted in the prostatic parenchyma in an array around the fiber tip. Study end-points included the clinical (urological), serological, radiological (TRUS, MRI) and pathological assessments of the treated prostates.

After the laser fiber and the thermocouples were correctly positioned in the prostate, the 5 min laser treatment was begun. Laser treatments were performed in 12 dogs at 2, 5 and 8 watts using an Nd:YAG and in 6 dogs using a diode laser at comparable wattage $(2,5$, 7 watts). Prostates were harvested at 7 or 60 days post-treatment.

\subsubsection{Treatment and clinical outcome of ILC}

At the start of laser treatment, an echogenic region rapidly developed that could be seen via TRUS to spread elliptically from the fiber end. The thermal injury was localized, with 
the highest temperature at the tip, and declining with distance from the tip. The thermal effect spread laterally until temperatures approached normal. The zone of raised temperature approximated the zone of hyperechogenicity seen on TRUS.

Urethral and perirectal temperatures never exceeded $66^{\circ} \mathrm{C}$. The laser tip was documented as properly placed in the center of a prostate lobe (with diameter of $\geq 1.75 \mathrm{~mm}$ ), and the urethral and perirectal temperatures never exceeded $40^{\circ} \mathrm{C}$. Maximum parenchymal treatment-temperatures were achieved in-line, and in-front of the laser tip. Maximal temperature for the 5-8 watt treatments often approached $100^{\circ} \mathrm{C}$ in this region. There was a marked treatment effect (lesion size) difference between 2 and 5 watts; however, there appeared to be minimal treatment-effect difference between 5 and 8 watts. There was good correlation between the treatment-effect produced by the Nd:YAG and diode lasers when similar wattage was used.

All dogs that were designated to survive the treatment did so with only minor acute urinary voiding complications. Transient dysuria and hematuria were observed in some dogs immediately following treatment. TRUS and MRI both proved capable of accurately assessing treatment effect.

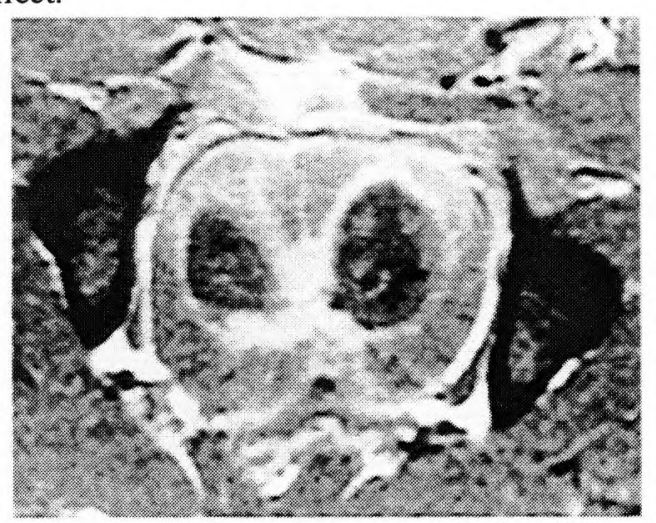

Figure 13. Magnetic resonance image of a canine prostate 60 days following ILC treatment consisting of 5 Watts $/ 5$ minutes in the left lobe and 8 Watts $/ 5$ minutes in the right lobe. The central dense regions in each lobe represent necrosis and/or hemorrhage. Although the treatments were well-localized to the center of each lobe and did not tear the capsule, the 8 Watt treatment encroached on the prostatic urethra. Although this dog retained the ability to urinate immediately following treatment, he experienced significant hematuria and dysuria for 3-5 days.

\subsubsection{Gross and histopathology results of ILC}

The acute ( 7 day) lesions were characterized by focal hemorrhage, a mixed-inflammatory infiltrate and parenchymal atrophy bordering cystic and/or non-cystic necrosis. The noncystic damage was characterized by fibrinoid necrosis of blood vessels, hemorrhage and degenerate parencymal cells that often retained the normal (glandular) architectural pattern, despite the fact that these cells would eventually slough. There was a moderate infiltration of neutrophils in the region immediately adjacent to the necrosis. Large zones of lymphoid infiltrate often effaced seriously-damaged parenchymal tissue. The nonnecrotic tissue immediately adjacent to the lesion was characterized by glandular atrophy and dilatation of ducts. 
Chronic laser lesions ( 60 days post-treatment) were almost always lined by continuous urothelium with an outer fibrous connective tissue rim. There was no evidence of hemorrhage or acute (suppurative/neutrophil) inflammation at this time; however, lymphocytic inflammation was extensive. Although there were areas of increased scaring/fibrosis adjacent to the primary cavitated-lesion, the glandular parenchyma around the lesion showed evidence of simultaneous parenchymal degeneration and regeneration. There was moderate fibrosis surrounding the cavity lesion; however, fibrosis was generally minimal in prostate parenchyma away from the lesion. Prostate size 60 days post-treatment was reduced (range 10-40\%) compared to pre-treatment measurements.

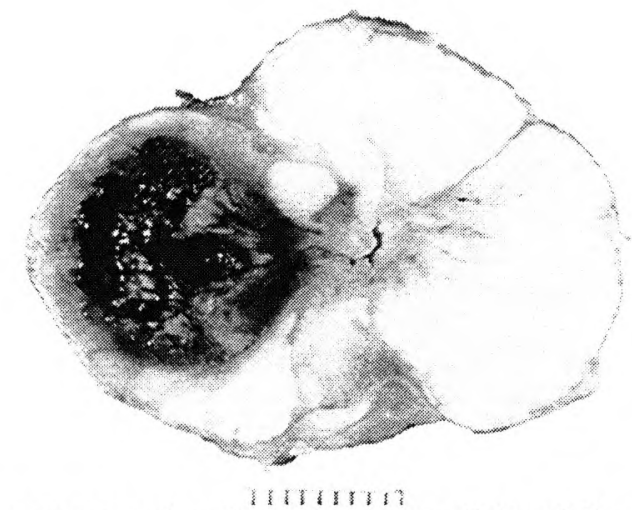

Figure 14. Axial/transverse section of a canine prostate 7 days following an 8 Watt ILC treatment of the left lobe. This treatment resulted in necrosis of a large portion of the treated lobe. The urethra and capsule were spared; however, the treatment effect was, perhaps, too close to these boundaries for ultimate safety. The dogs urinated without assistance during the 7 day post-treatment interval.

\subsubsection{Conclusions of ILC treatment modality}

The interstitial laser coagulation method produces a rapid, potentially well-controlled and safe treatment that can have a significant tissue ablation effect. An appropriate treatment guidance and monitoring modality such as TRUS, is essential for the effective use of ILC.

\subsection{Interstitial Cryotherapy (IC)}

In certain circumstances, cryotherapy has been successfully used to treat prostatic carcinoma, with a survival approximately equivalent to other treatment modalities. Cryotherapy has not been widely utilized due to local complications associated with the inability to monitor the freezing. Previously, the most important problem with cryosurgery has been defining the extent of the cryolesion. Transrectal ultrasoundguidance, however, has been shown to provide an accurate assessment of the cryolesion, and to provide the ability to adequately monitor the treatment thereby providing a mechanism for prevention of the complications associated with freezing of the urethra or the rectum. ${ }^{20}$

A number of open ${ }^{21}$ and percutaneous cryotherapy ${ }^{22}$ clinical studies for the treatment of prostate carcinoma have now been completed. Patient survival appears to be comparable to radical prostatectomy. However, morbidity including urethrorectal fistula (3\%); post- 
operative epididymitis (2\%); impotence (38-60\%); and incontinence (15\%) is equal to or greater than that following radical prostatectomy.

The subsequent development of transrectal ultrasound (TRUS) imaging and the ability to observe the cryo lesion during treatment should permit more accurate delivery of the cryotherapy with less morbidity. Frozen prostate has different acoustic properties that allow visualization of the freezing process. In addition, the cryo probes may be placed under ultrasound guidance using a percutaneous trans-perineal approach, and eliminating the need for an open surgical-procedure.

Petersen, et al. ${ }^{23}$ while studying post-cryotherapy biopsies, found that an increased level of inflammation correlated with improved patient outcome and tumor differentiation.

The objective of our IC study was to evaluate real-time ultrasound guidance in the production of 6 cryotherapy lesions in 4 canine prostates. Subacute and chronic histological effects of local cryosurgery on the prostate were evaluated at 6 and 14 weeks post-treatment using TRUS and histomorphometry/histomorphology.

\subsubsection{Methods of IC}

Immediately following standard anesthesia and surgical laparotomy preparation, a Foley catheter was positioned within the prostatic urethra. The catheter was equipped with 4 thermocouples spaced $1 \mathrm{~cm}$ apart to monitor transurethral temperatures. Using direct visualization and ultrasound guidance, additional temperature probes were placed just under the prostatic capsule near the rectum, bladder, and other adjacent tissues that might be at risk. Two dogs received one treatment to each lobe of the prostate (bilateral treatment), while the other 2 dogs had one lobe treated (unilateral treatment). Each cryo lesion was treated identically using a single, freeze/thaw cycle.

Under visual and transrectal ultrasound guidance, a custom-designed $3 \mathrm{~mm}$ (O.D.) cryo probe was inserted into the surgically exposed prostate lobe. Liquid nitrogen was delivered to the cryo probe using a Cryogenic Technology system (LCS 2000, Derbyshire, England). The freezing process was monitored using transrectal ultrasound, permitting real-time monitoring of the ice-ball formation. Each treatment consisted of a 6-10 min freeze-cycle (mean $8 \mathrm{~min}$ ) which was followed by a thawing period of approximately 15 minutes. The freezing cycle was discontinued when one of the urethral or subcapsular temperature probes approached $0^{\circ} \mathrm{C}$, or when the ice ball was observed to reach the prostatic capsule on ultrasound imaging. Thermocouple temperature measurements were simultaneously recorded at various locations by an IBM PC-based multi-channel computer data-acquisition system. After thawing, the cryo probe and thermocouples were removed. Following treatment, a urethral stint was placed in each animal to maintain patency of the prostatic urethra.

Temperatures were measured from within the prostatic urethra and at multiple subcapsular locations within the prostate. Treatment of an individual lobe was initiated at time 0 min followed by a delay as the liquid nitrogen first cooled the cryo probe, and then as the tissue-freezing progressed toward each of the thermocouples. There was also a delay noted after the freezing was discontinued as the temperature continued to drop for a few minutes after the liquid-nitrogen was turned off. The temperature data demonstrated the focal nature of the cryo-treatment. Core temperatures were recorded until the ice-ball approached the thermocouple, at which time a precipitous temperature drop was 
recorded. The temperatures in untreated areas (e.g., the untreated lobe) of the prostate remained at core body-temperature.

\subsubsection{Treatment and clinical outcome of IC}

One of the 6 prostate lobes treated, developed an abscess that was culture-negative. This animal had a mild clinical course, likely due to prophylactic antibiotic treatment, and survived without incident until the pre-determined 6-week end-point; however, that lobe was not evaluated. The other 3 dogs tolerated the treatments extremely well with virtually no morbidity.

Transverse-plane prostate TRUS images of both the 6 and 14 week end-points were obtained. TRUS imaging allowed easy observation of the treatment-induced cavitation urethra, prostate capsule and rectal wall. This finding is critical for the safe and accurate use of interstitial cryotherapy. The dimensions of the cavitary TRUS lesions corresponded precisely to the lesions observed histologically.

\subsubsection{Gross and histopathology results of IC}

Six weeks post-cryotherapy revealed a cavitary lesion lined by normal transitional cell urothelium. The cavity lesions, at this time and dose end-point, had volumes of approximately $1 \mathrm{~cm}^{3}$ and were surrounded by a 5-7 $\mathrm{mm}$ region that was devoid of normal prostatic glandular tissue and contained lymphocytes, hemosiderin-containing macrophages, and early fibrosis. Focal regions of glandular hyperplasia were widely scattered around the cavitary lesion. There were no polymorphonuclear cells to suggest acute inflammation. However, there were well-defined foci of lymphocytes and plasma cells distributed throughout the prostate (treated and untreated lobes) suggesting a chronic inflammatory or immune response. In prostates with a unilateral treatment, the aggregates of lymphocytes were larger and more numerous in the treated hemisphere.

At 14 weeks the urothelial-lined cavitary lesion remained, but when compared to the 6week dogs, there was more mature fibrosis and/or fibromuscular stroma surrounding the cavitation. Foci of glandular hyperplasia were present close to the wall of the cavity. In addition, there was regeneration of glandular tissue and shrinkage of the glandular area observed at 6 weeks. Periurethral fibrosis was not increased in the 14-week dogs. Table I demonstrated a mild increase in FCT stroma and a corresponding decrease in glandular parenchyma at both 6 and 14 weeks.

Table I. This table demonstrates the histological quantitation of relative tissue components of the interstitial cryotherapy-treated prostates at 6- and 14-week end-points.

\begin{tabular}{|l|c|c|c|c|}
\hline & $\begin{array}{c}\text { Untreated } \\
\text { (6-wk post-treat) }\end{array}$ & $\begin{array}{c}\text { Treated } \\
\text { (6-wk post-treat) }\end{array}$ & $\begin{array}{c}\text { Untreated } \\
\text { (14-wk post-treat) }\end{array}$ & $\begin{array}{c}\text { Treated } \\
\text { (14-wk post-treat) }\end{array}$ \\
\hline FCT & $20 \%$ & $33 \%$ & $23 \%$ & $27 \%$ \\
Muscular stroma & $11 \%$ & $8 \%$ & $13 \%$ & $13 \%$ \\
Glandular & $40 \%$ & $37 \%$ & $37 \%$ & $32 \%$ \\
Void/Lumen & $16 \%$ & $13 \%$ & $23 \%$ & $17 \%$ \\
Inflammation & $13 \%$ & $10 \%$ & $3 \%$ & $12 \%$ \\
\hline
\end{tabular}


Like other focal prostate ablation-treatments, cryotherapy induced a marked, multifocal lymphocytic-response that appeared to morphologically, most-closely-resemble nodular lymphoid hyperplasia and/or an immune-mediated response. This reaction might be clinically significant, since $\mathrm{Gage}^{24}$ asserted that the treatment of carcinoma of the prostate with cryotherapy could lead to a potentiation of immunologic defenses.

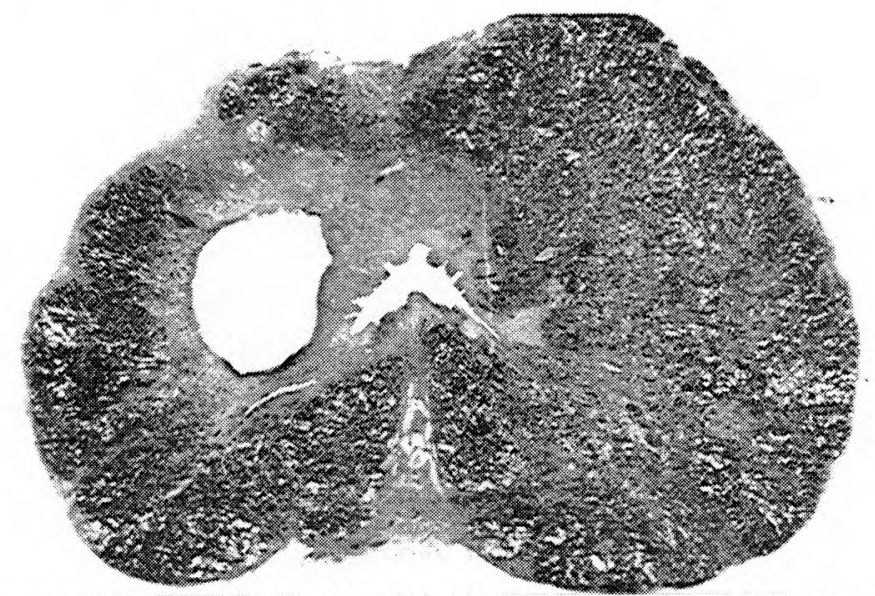

Figure 15. Transverse/axial section of canine prostate gland 14 weeks post-cryotherapy. The cavitated region in this gland was approximately $1 \mathrm{~cm}^{3}$ with a complete urothelial lining. The cavity was surrounded by fibrous connective tissue and scattered lymphocytic infiltration. A zone of parenchymal loss, fibrosis and mild lymphoid-infiltration was also seen extending from the capsule to the cavity. The upper-left region in this prostate (adjacent to the cavity), was penetrated by the cryotherapy probe. The treated hemisphere was notably smaller than the non-treated hemisphere.

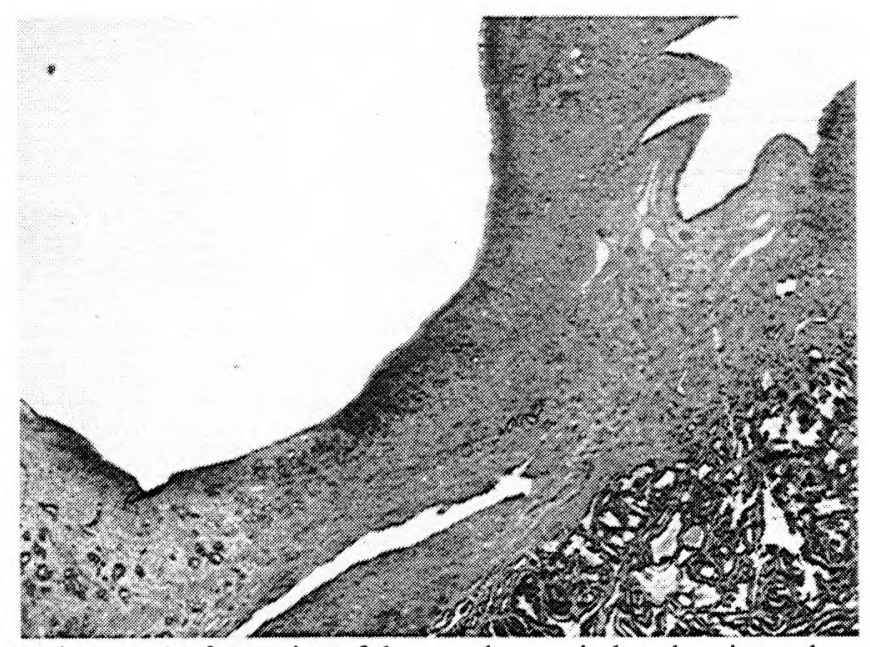

Figure 16. Photomicrograph of a portion of the cryotherapy induced cavity and surrounding tissue. As previously described, the immediate boundary of the cryotherapy lesion is largely composed of fibrous connective tissue (FCT), occasional atrophied glands and scattered inflammation. The relatively abrupt change from FCT/parenchymal atrophy, to normal parenchyma (lower right), and a portion of the undamaged urethra (upper right) can also be seen. (H\&E; 10x mag.) 


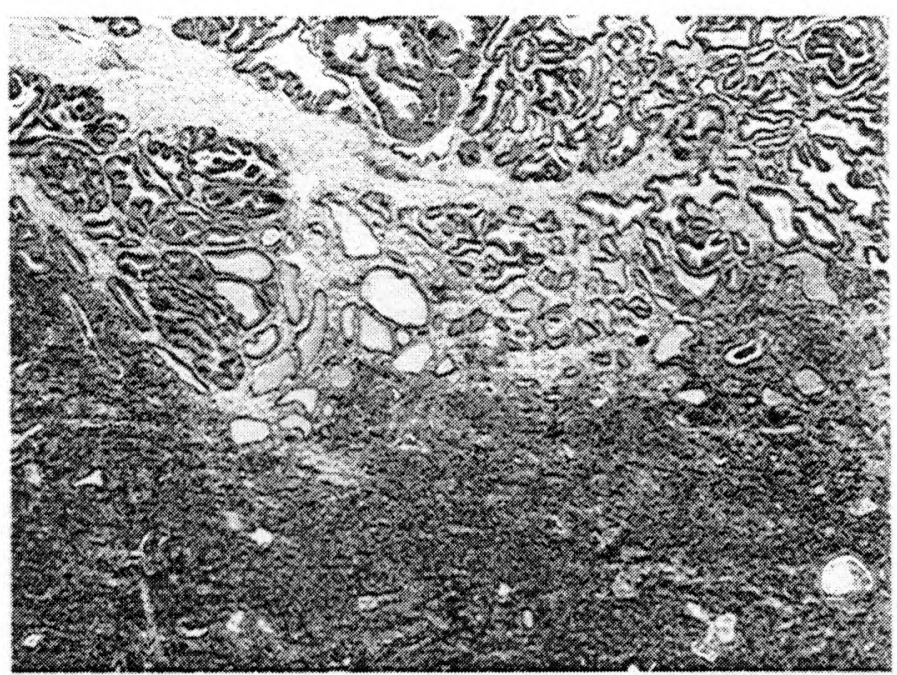

Figure 17. Photomicrograph of parenchymal effacement by lymphocytic inflammation (lower onehalf of photo). Although this pattern of parenchymal loss and lymphocytic replacement was not the only pattern of inflammation, it often occurred in regions of significant treatment-induced damage. (H\&E; 10x mag.)

\subsubsection{Conclusions of the IC treatment modality}

Ultrasound-guided percutaneous transperineal cryosurgery shows promise as a less invasive and possibly less-morbid technique for treating some prostate cancers. It has previously been shown that the ultrasound-defined border corresponds well with the histopathologic cavitary lesion in the acute phase. We have extended these findings by examining the cryosugical lesion at relatively chronic stages ( 6 and 14 weeks). Our cryoinduced lesions were not intended to ablate the entire prostate, but rather to ablate a specifically targeted region. TRUS and whole organ histologic mounts of the canine prostate permitted detailed examination of the region surrounding the cavitary cryolesion, the evolution of the lesion over time, and the direct comparison of TRUS and histologically-imaged treatment effects. Although the primary cavitation lesion was normally surrounded by a reasonably large rim of fibrous connective tissue at the 14 week end-point, alternatively the 6 week post-treatment lesions showed abrupt changes between cavitation and healthy tissue. This suggested that the cryotherapy resulted in acute death or survival of cells at a specific region of treatment.

\section{CONCLUSIONS}

\subsection{Overview}

In spite of significant differences in the types of energy/procedures used in the studies, the pathophysiologic reactions in the prostate were often very similar. The prostate, like other tissues, has limited injury response pathways. Therefore, in general, these studies have shown that the chronic effects of focalized extreme-heat and -cold are fundamentally very similar to surgical removal of segments of prostate tissue. Therefore, while our studies have shown marked tissue response similarities for the various treatment modalities, ease of use and potential tissue complications/risks and the need for real-time 
and accurate applicator/application imaging are variable and need to be addressed separately for the safe and efficacious use of each modality.

In summary, it appears that the prostate treatment technique studies here are clearly more invasive, yet more effective, than medical BPH/prostate cancer treatments, and less invasive, but less effective than major surgical procedures designed to treat the same diseases.

\subsection{Safety}

Transurethral applicators and applications are generally less complicated and less invasive to perform, respectively. Many of those applications can be initiated through relatively straight-forward catheterization/direct visualization procedures. The use of a bladder locator-balloon often allows accurate and easy placement in the designated region of the prostatic urethra. The risk of these types of procedures lies primarily in localization of the applicator and contour of the treatment procedure.

Interstitial applications such as cryotherapy, cooled microwave hyperthermia, and laser ablation of the parenchyma are potentially less toxic to the prostatic urethra (if performed properly); however, they pose the real possibility of damage to the capsule and/or to the tissue adjacent to the prostate such as the rectum. It appears that the interstitial applicators will almost certainly require accurate real-time imagery for proper placement and accurate safe-treatment. On the other-hand, lumen-based treatments such as TURT are generally safe to non-urinary organs, but carry a risk of sphincter and nerve damage.

\subsection{Imaging}

Although we have successfully used MRI to assess the acute and chronic tissue effects seen in the prostates we treated, this modality is not currently available for routine realtime treatment monitoring. Therefore, standard urethroscopy/fluoroscopy and transrectal ultrasound (TRUS) are the most useful tools for proper positioning of prostate treatment applicators. However, of these modalities, only TRUS is appropriate and readily available for real-time monitoring of applicators such as focal, thermal and laser ablation and cryotherapy. In-spite-of the usefulness of TRUS, it appears to us that more imaging research targeted to the individual application, is necessary for improvements in the safety and efficacy of the minimally-invasive techniques discussed here.

\subsection{Efficacy}

As previously mentioned, all of the prostate treatment applications we detailed here, with the possible exception of radio-frequency balloon-thermotherapy, are designed to produce focal necrosis. While we assume focal necrosis is likely to be an efficacious nonsurgical treatment for bulky BPH and focal prostate cancer, our studies suggest that these treatments are only able to produce the appropriate effect if the treatment imaging and pathophysiology are well understood. Additionally, it remains unclear if focal ablation of prostate parenchyma translates to improved urinary performance scores over the longterm. 
Matching the Energy Source to the Clinical Need / 543

\subsection{Immunology}

Our studies support the previously published concept ${ }^{20}$ that cytolytic damage to the prostate (canine and human) evokes a profound and possibly pathognomonic immunologic/inflammatory reaction. Our studies have shown that this reaction is almost entirely composed of T-cells (probably early, uncommitted T-cells) at the time of assessment. It is yet unclear what role this response may play in the long-term effectiveness of a BPH parenchymal ablation technique or on the success of a cancer treatment procedure.

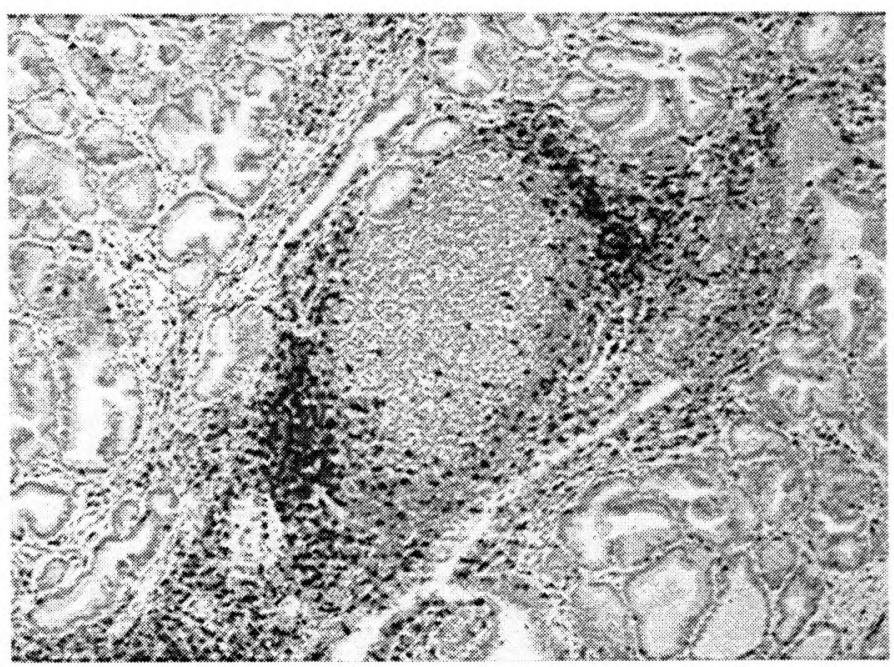

Figure 18. Photomicrograph of a lymphoid nodule in the parenchyma of a canine prostate gland 6 months following TUMT. CD3 staining of lymphocytes bordering the lymphoid nodule suggests that these T-cells are now involved in transducing antigen-recognition signals into the cytoplasm of $\mathrm{T}$-cells, and involved in regulating the cell surface expression of the T-cell antigen receptor. The unstained lymphocytes bordered by the stained cells are T-cells not yet committed to a specific Tcell subset. These types of lymphoid nodules were present to varying degrees in virtually all canine prostates treated using the 6 modalities presented. (H\&E; 10x mag.) 


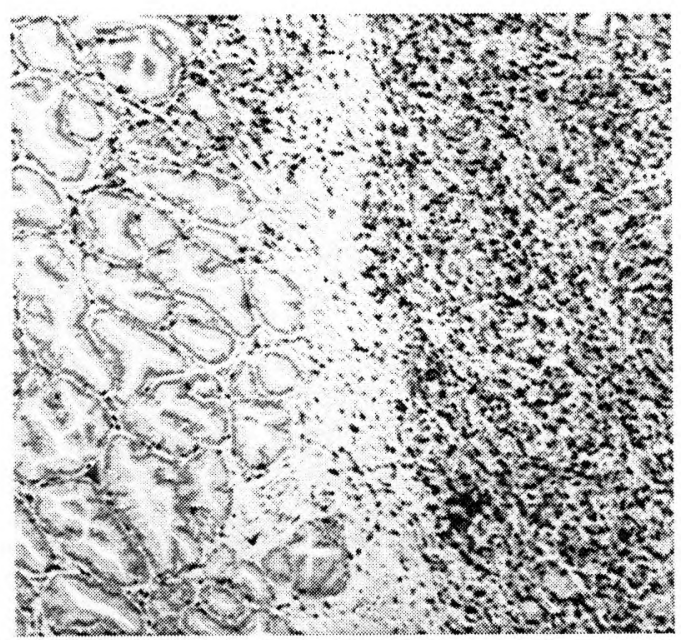

Figure 19. Photomicrograph of CD3 T-cell lymphocytes occupying a very well defined segment of prostate parenchyma 6 months following treatment with cryotherapy. This type of segmental reaction including parenchyma effacement, which is heavily populated by $\mathrm{CD} 3$ positive

lymphocytes, was seen to varying degrees in virtually all canine prostates treated by the authors. This pattern of reaction, which is often wedge-shaped (urethra to capsule), can be very well-defined and is not directly associated with the treatment lesion. Although not proven, these patterns of tissue damage/reaction would appear to be associated with a vascular pattern. CD20 staining for Bcells was largely negative in prostates we treated. (H\&E; 10x mag.)

\section{ACKNOWLEDGMENTS}

This work was supported in part by NIH grant CA57037; Microvasive/ Boston Scientific; and American Medical Systems.

\section{REFERENCES}

1. Guess HA. The epidemiology and natural history of benign prostatic hyperplasia. IN Handbook on Benign Hyperplasia, GD Chisholm (ed). Merck \& Co, NJ, pp. 1-18, 1994.

2. Nielson SW, Kennedy PC. Tumors of the genital system. IN Tumors in Domestic Animals, JE Moulton (ed). University CA Press, Berkeley, pp. 491-495, 1990.

3. Lee, Torp-Petersen, Siders. The role of TRUS in early detection of prostaate cancer. CA - A Cancer Journal for Clinicians. American Cancer Society, NY, pp. 347-350, 1989.

4. Habib FK. Pathogenesis of benign prostatic hyperplasia. IN Handbook on Benign Hyperplasia, GD Chisholm (ed). Merck \& Co, NJ, pp. 19-31, 1994.

5. Kaplan SA, Dowd JB. Non-pharmacologic alternative therapy in the management of benign prostatic hyperplasia. IN Handbook on Benign Hyperplasia, GD Chisholm (ed). Merck \& Co, NJ, pp. 115-136, 1994.

6. Mebust MK. Transurethral surgery. IN Campbell's Urology, PC Walsh, et al., (eds). Saunders, Philadelphia, pp. 2911-2922, 1992.

7. Wingo PA, Tong T, Bolden S. Cancer statistics 1995. CA - A Cancer Journal for Clinicians. American Cancer Society, NY, pp. 8-30, 1995.

8. Hanno PM. Carcinoma of the prostate. IN Current Urologic Therapy, EJ Seidmon, PM Hanno (eds). WB Saunders, Philadelphia, pp. 384-386, 1994. 
9. Heaton P. Radiofrequency thermal ablation of the prostate: The TUNA technique. Techniques Urology, 1(1):3-10, 1995.

10. Dawkins GP, Harrison NW, Ansell W. Radiofrequency heat treatment to the prostate for bladder outlet obstruction associated with benign prostate hyperplasia: A 4-year outcome study. British J Urology, 79(6):910-914, 1997.

11. Yoshida O, Terai A, Shichiri Y, et al. Transurethral radiofrequency thermotherapy for benign prostatic hyperplasia: Preliminary clinical results. International J Urology, 3(6):454-458, 1996.

12. D' Ancona FC, Albers YH, Klemeney LA, et al. Can histopathology predict treatment outcome following high-energy transurethral microwave thermotherapy of the prostate? Results of a biopsy study. Prostate, 40(1):28-36, 1999.

13. Eliasson T, Abramsson L, Damber JE. Importance of thermal dose and antenna location in transurethral microwave thermotherapy for benign prostatic hyperplasia. $\mathrm{J}$ Endourology, 12(6):581-589, 1998.

14. D'Ancona FC, Francisca EA, Hendriks JC, et al. The predictive value of baseline variables in the treatment of benign prostatic hyperplasia using high-energy transurethral microwave thermotherapy. British J Urology, 82(6):808-813, 1998.

15. Barbalias CA, Liatsikos FN. Transrectal microwave hyperthermia for patients with benign prostatic hyperplasia. International J Urology, 5(2):157-162, 1998.

16. Stawarz B, Zielinski H, Szmigielski S, et al. Transrectal hyperthermia as palliative treatment for advanced adenocarcinoma of the prostate and cell-mediated immunity. Urology, 41(6):548-553, 1993.

17. McNicholas TA, Steger AC, Brown SG. Interstitial laser coagulation of the prostate: An experimental study. British J Urology, 71:439, 1993.

18. Littrup PJ, Lee F, Borlaza GS, et al. Percutaneous ablation of canine prostate using transrectal ultrasound guidance: Absolute ethanol and Nd:YAG laser. Invest Radiol, 23:734-739, 1988.

19. Perlmutter AP, Muschter R. Interstitial laser prostatectomy. Mayo Clinic Proceedings, 73(9):903-907, 1998.

20. Onik G, Porterfield B, Rubinsky B, et al. Percutaneous transperineal prostate cryosurgery using transrectal ultrasound guidance: Animal model. Urology, 37:277$281,1991$.

21. Kunit G. Open-perineal-cryosurgery in carcinoma of the prostate - a possible curative alternative. Urology Research, 14:3-7, 1986.

22. Miller RS, Cohen JK, Merlotii LA. Percutaneous transperineal cryosurgical ablation of the prostate for primary treatment of clinical stage $\mathrm{C}$ adenocarcinoma of the prostate. Urology, 44(2):170-174, 1994.

23. Peterson DS, Milleman LA, Rose EF, et al. Biopsy and clinical course after cryosurgery for prostate cancer. J Urology, 120(3):308-311, 1978.

24. Gage AA. Cryosurgery in the treatment of cancer. Surgery Gynecol. Obstetrics, 174(1):73-92, 1992. 